\title{
Herbovigilance at the Crossroads in India: Prospects and Some Introspects
}

\section{Raja Chakraverty*}

ICMR Research Fellow, Department of Pharmacology, Institute of Post Graduate Medical Education and Research, Kolkata, India

*Corresponding Author: Raja Chakraverty, ICMR Research Fellow, Department of Pharmacology, Institute of Post Graduate Medical Education and Research, Kolkata, India.

Received: October 15, 2019; Published: November 01, 2019

DOI: $10.31080 /$ ASPS.2019.03.0430

Pharmacovigilance (PV) activities have indeed come a long way in India since the inception of national policy facilitating the transition from the erstwhile National Pharmacovigilance Program to ushering the genesis of the Pharmacovigilance program of India (PVPI) back in 2010. Broadly, this science and practice encompasses activities related to the detection, assessment, understanding and prevention of adverse drug reactions (ADR) or any other possible drug-related problems including the much neglected case of herb-drug interactions. In the recent past, however, its purview has been widened to include herbal, traditional and complementary medicines (AyUSH) with the goal to comprehensively detect, assess, and understand adverse reactions from herbal products with the unanimous goal of preventing occurrence of adverse effects among end-users. The established methods for conducting herbovigilance is similar to PV and includes but are not limited to- spontaneous reporting, cohort report monitoring and targeted spontaneous reporting from adverse drug monitoring centres (AMC) scattered all across over this nation and collated and analysed at the CDSCO, Ghaziabad and ultimately at the Uppsala Monitoring Centre (WHOUMC) based in Sweden. Signal detection and causality assessment of reports generated from herbal products assume a great significance owing to its use by a large proportion of fellow citizens in both rural and urban demography and thus its role in reporting of rare herbal medicine induced disorders that often goes unnoticed. The literature search conducted presenting this commentary was based on extensive topic related search of contemporary scientific articles and complementary review of bibliographies from selected publications on the subject of Herbovigilance and its chronological evolution till date. The promotion of systematic and rational use of drugs and shift of the paradigm from drug safety to patient safety requires the reporting of adverse events possibly caused by herbal and traditional medicines with their mechanistic underpinnings. Proper reporting of suspected adverse drug reactions from herbal medicine practitioners in Ayurveda, siddha and other Ayush disciplines has, therefore, assumed a greater role in the holistic outlook of therapeutics of today and requires proper and careful vetting prior to guideline based implementation from stakeholders in the healthcare sector (practitioners, pharmacists and nurses). Thus, in summary this editorial attempts to stress on the need for nurturing our national herbovigilance activities and creation of many more resource centres which is quite essential to build up reliable information database much like Vigibase based on data from the Indian network regarding the safety of herbal medicines to boost confidence about their safety making it at par with the system of modern medicine in terms of the credible evidence available in public and legislative domains.

\section{Volume 3 Issue 12 December 2019 (C) All rights are reserved by Raja Chakraverty.}

